\title{
Advances in SDD-based EDS and Comparisons to WDS for Light Element Sensitivity
}

\author{
Keith Thompson ${ }^{1}$
}

1. Thermo Fisher Scientific, Madison, WI.

Silicon Drift-based Energy Dispersive Spectroscopy (EDS) X-ray detectors have made strong advances in light element sensitivity over the last several years. 25 years ago most detectors still utilized Be (or similar performing) windows that prohibited meaningful transmission of X-rays below $1000 \mathrm{eV}$. The advent of light element polymer windows enabled detector sensitivity to C. Successive improvements in window construction resulted in light element sensitivity improvements from B to Be and finally to Si-L and Al-L. Example spectra are shown in Fig. 1.

Unfortunately, these "sensitive to element" specifications rely entirely on the analysis of pure element standards. The complete removal of the light element window demonstrates the misleading nature of the "sensitive to element" specification for comparing window technologies when pursuing the ultimate in soft X-ray detection, see Fig. 2. First, a comparison of metallic Be X-ray spectra acquired with and without an entrance window shows a 10x improvement in intensity with the removal of the window. This is followed by a comparison of EDS X-ray spectra of $2 \% \mathrm{~B}$ in glass, with the B, Si-L and P-L peaks clearly visible with the windowless design and effectively undetected with a light element window. Finally, analysis of a Lithium Phosphor Sulfide (LPS) sample solidifies the clear superiority of a windowless design when seeking true soft X-ray detection.

With these advances in soft X-ray detection by EDS, is WDS still relevant? Mapping the distribution of $2 \% \mathrm{~B}$ in steel, Fig. 3, provides insight into the role that signal-to-noise can play in mapping the distribution of trace light elements. The backscattered electron image indicates two regions of elemental interest. The windowless EDS X-ray element map confirms this assessment with the presence of $2 \% \mathrm{~B}$ in an FeCr matrix. The WDS moves beyond these analyses to identify a $3^{\text {rd }}$, zoned region of $1.2 \% \mathrm{~B}$ within the FeCr matrix. This level of detail, examining the X-ray spectral overlays of WDS and EDS, is only possible with the order of magnitude improvement in signal-to-noise possible with modern WDS.

This talk focuses on the historical improvement of EDS detectors over the years with a look forward to future improvements, a consistent comparison to the continued improvements of WDS for soft X-ray detection and an analysis of the role software algorithms can play in extracting the meaning of subtle changes in the $\mathrm{x}$-ray spectrum on the distribution of trace light elements, including Li, within a material. 

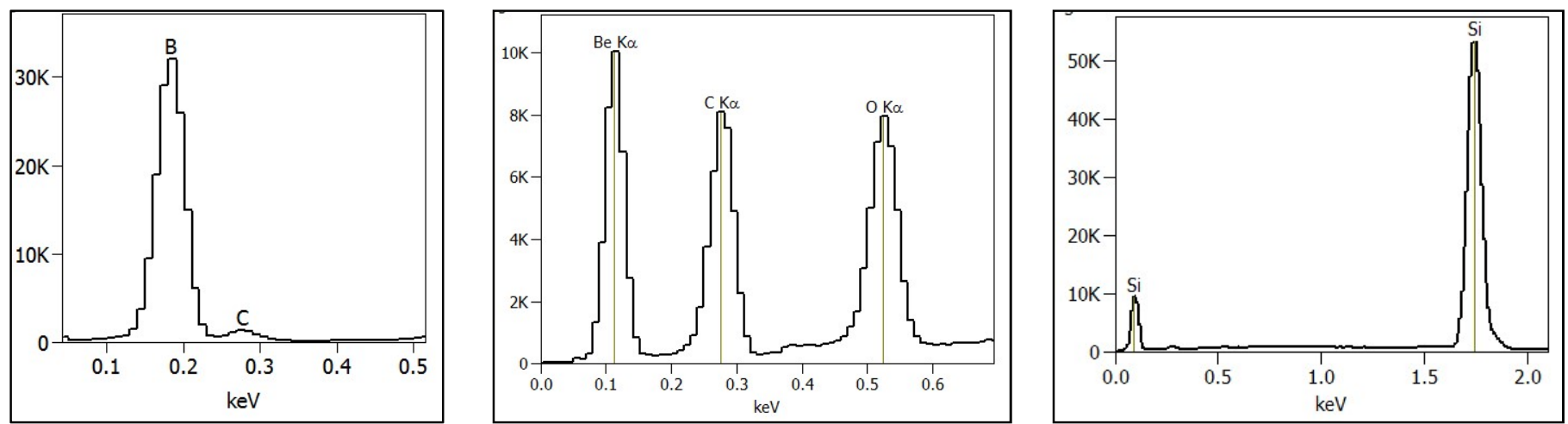

Figure 1. EDS X-ray spectrum of (Left) metallic B; (Center) metallic Be; and (Right) Si.
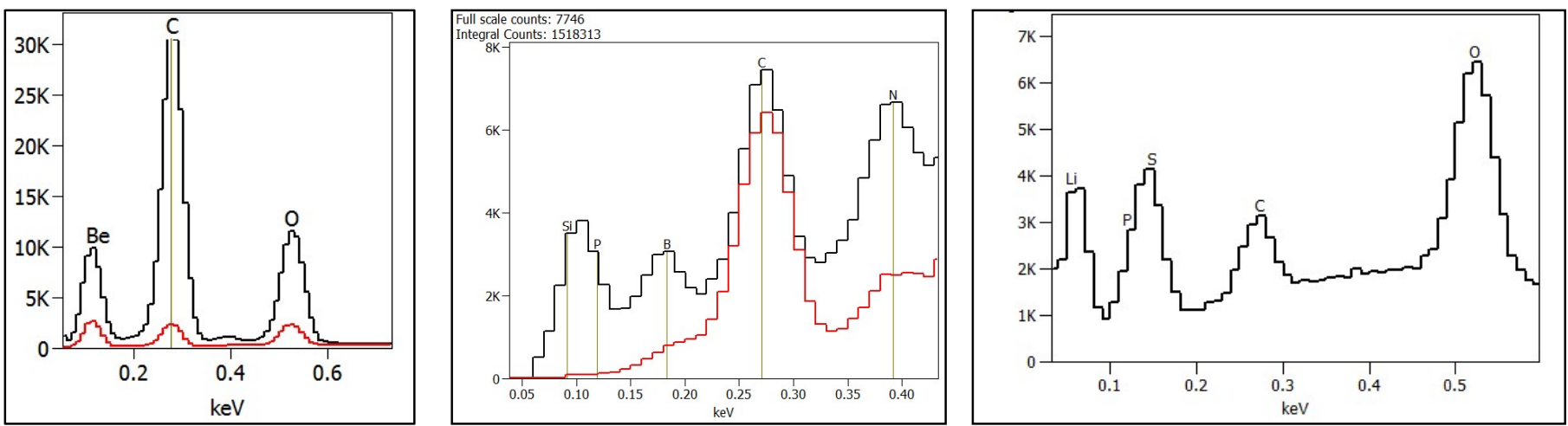

Figure 2. EDS X-ray spectrum of (Left) metallic Be - window (red) vs. windowless (black); (Center) 2\% B in glass - window (red) vs. windowless (black); (Right) Lithium Phosphor Sulfide - windowless.

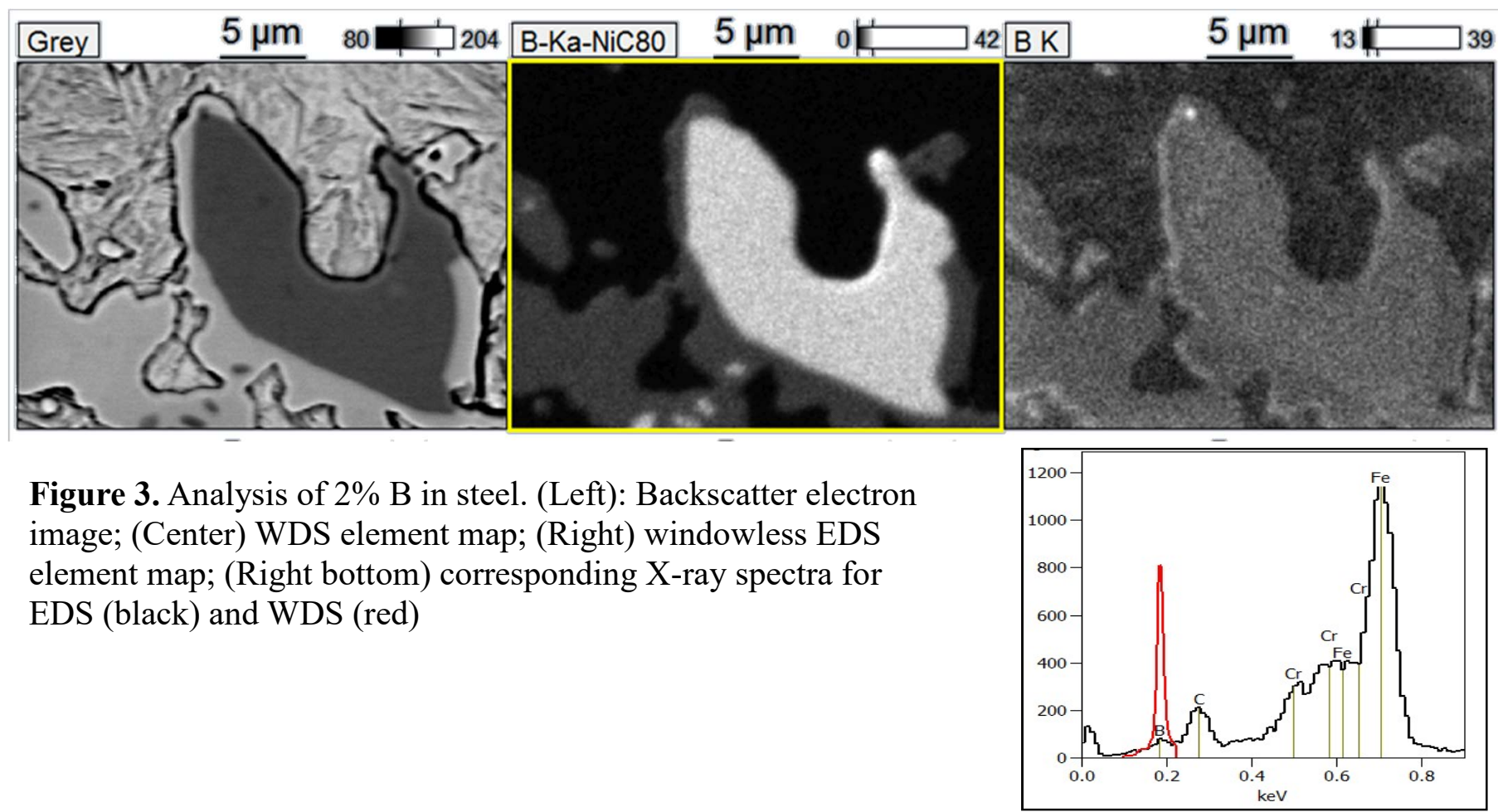

